\title{
ANÁLISIS DE LA COMPETENCIA LINGÜÍSTICA EN PRIMARIAA TRAVÉS DE LAS TIC
}

\section{ANALYSIS OF PRIMARY LANGUAGE COMPETITION THROUGH ICT}

\author{
Francisco Manuel Marchal López \\ fmmarchal@gmail.com \\ Cristina Sánchez Romero \\ csanchez@edu.uned.es \\ Ana María Martín-Cuadrado \\ amartin@edu.uned.es
}

\begin{abstract}
UNED. Facultad de Educación, Departamento de Didáctica, Organización Escolar y Didácticas Especiales
\end{abstract}

Resumen: Este artículo muestra el uso de las TIC para la mejora de la competencia lingüística de los estudiantes de Educación Primaria. Desde un diseño cuasi-experimental, la muestra conformada por 34 estudiantes de tercer ciclo de Educación Primaria de diferentes centros educativos en la comunidad andaluza, se ha dividido en dos grupos, grupo de control y grupo experimental, con la medición de la variable competencia lingüística a través de un cuestionario pretest-postest $(?=, 821)$. A través del análisis estadístico para muestras independientes $T$ de Student, los resultados muestran diferencias significativas en la variable de competencia lingüística $(t=2,506 ; p=0.017)$ obteniendo un mayor valor en el grupo experimental. En conclusión, se mejora la competencia lingüística con las TIC en las rutinas aprendizaje.

Palabras clave: TIC, educación primaria, competencia lingüística.

Abstract: This article shows the use of ICTs for the improvement of Primary Education students' linguistic competence. From a quasi-experimental design, the sample made up of 34 students of the third cycle of Primary Education from different educational centers in the Andalusian community, has been divided into two groups, the control group and the experimental group, with the measurement of the linguistic competence variable through a pretest-postest questionnaire (? =, 821). Through statistical analysis for independent Student's $T$ samples, the results showed significant differences in the linguistic competence variable ( $t=$ 2.506; $p=0.017)$, obtaining a higher score in the experimental group. In conclusion, the linguistic competence improves when the ICT is applied in learning routines.

Keywords: ITC, Primary Education, linguistic competence.

Píxel-Bit. Revista de Medios y Educación. No 53 Julio 2018. ISSN: 1133-8482. e-ISSN: 2171-7966. doi: http://dx.doi.org/10.12795/pixelbit.2018.i53.08 


\section{Introducción.}

La inclusión de las TIC en los procesos educativos, ya sean formales o informales es un estándar a tener en cuenta. Bajo el amparo de este argumento, la Consejería de Educación de la Junta de Andalucía, tiene como meta generalizar la incorporación de las TIC a la práctica docente a través del acceso de los centros a planes y programas educativos considerados de importancia estratégica, explicitados en la Ley 17/2017, de 10 de diciembre, de Educación en Andalucía. En esta línea, el nexo de unión que se establece entre competencia lingüística y uso de las tecnologías es vital y sintomático. En el desarrollo curricular de la etapa de Educación Primaria, se otorga una importancia al uso de las TIC para el desarrollo de la competencia lingüística al conceder a las tecnologías educativas el poder proporcionar conocimientos, destrezas y habilidades para la comprensión, la mejora de los procesos lectores, el uso de la expresión oral y escrita, etc. y por ende, en la totalidad y globalidad de la competencia comunicativa.

La utilización de las TIC han estado en continuo debate, la creencia de que las TIC ayudan a «enseñar mejor»y que los discentes «aprenden mejor» con las mismas ha sido analizada por diferentes autores (Area Moreira, 2005, 2010; Coll, 2009; Himle \& Woods, 2005; Macau, 2004). Es más, según algunos autores, las políticas educativas en el sistema educativo español, se enfrentan al reto de evitar la noción de que la simple presencia de TIC en un aula represente indiscutiblemente una mejora en la calidad de la educación (Alonso Cano, Romeu Fontanillas, y Guitert Catasús, 2014).
En esta línea, el estudio «Students, Computers and Learning: Making the Connection» (OECD, 2015)no solo cuestiona el valor de la TIC, sino que manifiesta que no hay correlación entre el uso de las TIC en la escuela y los resultados obtenidos. Es más, en países como Emiratos Árabes, Brasil, Chile y Colombia, muestran una correlación negativa entre tiempo de exposición del alumnado a las TIC y su bajo desempeño en pruebas escritas y lectura, donde se pone en juego el desarrollo de la competencia lingüística.

De forma antagonista, estudios realizados sobre la eficacia del programa Escuela 2.0 que se inició en el curso escolar 2009/2010, apuntaban que esta iniciativa no ha tenido los efectos didácticos deseados en el modo de organizar y gestionar los procesos de enseñanza-aprendizaje (Santiago Campión, Navaridas Nalda, y Repáraz Abaitua, 2013), y más concretamente en el desarrollo de la competencia comunicativa. En definitiva, no se observan mejoras evidentes en el aprendizaje de las áreas curriculares instrumentales, ni transformación significativa en las estrategias metodológicas del profesorado.

A la vista de estos resultados, parece claro que si bien hay alguna evidencia que confirma el impacto positivo de las TIC, éstas aún no parecen determinantes. Las conclusiones son obtenidas en circunstancias muy particulares no permitiendo realizar generalizaciones. Además, los estudios de gran escala, en la mayoría de las ocasiones, no permiten aislar el efecto neto del uso de las TIC y son analizados sólo una vez en el tiempo. «... a pesar de la cantidad y abundancia de investigación empírica sobre los procesos de incorporación de las TIC en las escuelas, 
todavía «los resultados de investigación... se caracterizan por inferencias poco convincentes e inconsistentes que socavan la generalización de conclusiones y reducen significativamente su impacto» (Maria Mama \& Hennessy, 2013).

Así pues, se entiende justificada la necesidad de seguir valorando la capacidad de las TIC para favorecer el desarrollo de la competencia lingüística.

El cuerpo del artículo estará mecanografiado a doble espacio. Estará dividido en apartados y subapartados, hasta el tercer nivel, numerados en arábigo. Tanto apartados como subapartados estarán escritos en minúscula y en negrita y se separarán entre ellos y los párrafos anteriores y posteriores, respectivamente, con un renglón en blanco.

Tanto las tablas, gráficos y figuras deberán aparecer numerados en arábigo (ejemplo: Tabla 1.), por orden correlativo para cada tipo y subtitulados. Deberán estar referenciados en el texto. No se admitirán imágenes que sean redundantes con las tablas, gráficos y/o figuras contenidos en el artículo. En las tablas el tamaño de letra utilizada será de 10 puntos.

\section{Marco teórico.}

En el marco legislativo actual, se destacan los aprendizajes que son imprescindibles para los estudiantes y que son importantes transferir del ámbito escolar a la vida cotidiana. En este sentido, se pone énfasis en una serie de competencias clave, recogidas en la normativa curricular andaluza, entre las que se integra la competencia en comunicación lingüística. De esta manera, se consagra como una de las competencias clave que ha de trabajarse en los centros educativos, y desde todas las áreas curriculares en Educación Primaria. Este hecho no es arbitrario puesto que la comunicación, especialmente la lingüística, es una herramienta básica de adaptación en los ámbitos cognitivo y socioemocional (Mariscal et al., 2007). La propia normativa curricular conceptualiza la competencia lingüística dentro del área de Lengua Castellana y Literatura, como «el conjunto de conocimientos, destrezas y actitudes necesarios para el uso de la lengua, como instrumento para expresión y comunicación, que posibilita la experiencia humana de la realidad y el pensamiento, en general.

Tanto la comunicación oral como la comunicación escrita se ha visto «atrofiada» por las tecnologías de la comunicación. La comunicación oral, desde hace décadas, se produce de forma diferida (telefonía móvil, aplicaciones digitales, televisión, soportes para la reproducción musical, etc.); quizás, la comunicación escrita se ha vista más ralentizada hasta la inclusión de internet en nuestra necesidad diaria contribuyendo a la inmediatez (al igual que la comunicación oral), a una mayor interacción y al uso exponencial de distintos códigos no verbales (emoticonos, imágenes...) Un ejemplo de este uso y del cambio que ha supuesto la inclusión de la digitalización en nuestras vidas lo han puesto de relieve (Nardocci \& Nascimento, 2012) al analizar las modalidades del discurso en foros digitales. Especialmente, la lectura, gracias al uso de nuevos soportes se ha sumado a cambios en las estructuras tradicionales, como el hipertexto, intertextualidad y el uso de otros lenguajes combinados: imágenes, sonidos o vídeos, para lo que se hace necesaria una formación específica. Por consiguiente, «...a la lectura lineal del libro tradicional le sucede 
el hipertexto como estructura básica del discurso: numerosos fragmentos escritos, breves, monotemáticos y autónomos, se conectan entre sí con enlaces a modo de red o entramado (Cassany, 2005, 2008; Ramírez Leyva, 2001)y frente a las ilustraciones de los textos clásicos encontramos las imágenes, los gráficos, los sonidos o los vídeos que acompañan y completan los textos digitales» (Ramírez, Renés y Delgado, 2014, p.347).

De esta manera, la adquisición de la competencia comunicativa en el actual ecosistema digitalizado, ha multiplicado de una manera inimaginable la compleja diversidad de los lenguajes y de los canales de la comunicación humana (Gracida y Lomas, 2011), requiriendo una atención explícita a muchos de los aspectos involucrados en el uso integrado de las TIC dentro de los procesos de enseñanza aprendizaje. En esta línea, las personas que tienen competencias digitales tienen la capacidad en dimensiones comunicativas (García Ruiz, Ramírez García, y Rodríguez Rosell, 2014), y por ende, deben obtener mejores resultados en la competencia lingüística.

Para la medición de la variable competencia lingüística, objeto de este estudio, la Evaluación Diagnóstica de Andalucía (EDA) ha llevado a cabo el diseño, elaboración e implementación de pruebas escritas con carácter homologado con el objetivo de obtener resultados contrastables. Estas pruebas permiten cubrir de forma exhaustiva el contenido objeto de evaluación (Consejería de Educación, 2007).

\section{Método.}

Para la realización de este estudio se ha optado por un diseño pre-post con grupo control que nos permitiera evaluar con precisión el impacto de la intervención basada en el uso de las TIC.

\subsection{Objetivo.}

Como señaló Underwood (2011, p.17) y con toda vigencia, «el momento actual requiere un alto en el camino y evaluar de forma objetiva lo que la tecnología puede y no puede ofrecer, realmente, hasta que se llegue a un punto de productividad en el que la tecnología cumpla con las metas realistas que proponemos». Para responder a esta hipótesis, analizaremos la relación entre la inclusión de las TIC y el desarrollo de la competencia lingüística. De esta manera, el objetivo de estudio propuesto sería el siguiente: analizar el impacto del uso de las TIC en la competencia lingüística en Educación Primaria.

\begin{tabular}{|l|l|l|}
\cline { 2 - 3 } \multicolumn{1}{c|}{} & \multicolumn{1}{c|}{ GRUPO CONTROL } & \multicolumn{1}{c|}{ GRUPO EXPERIMENTAL } \\
\hline N ${ }^{\circ}$ de alumnos & 16 & 18 \\
Edades & $10-12$ & $10-12$ \\
CEIP & Fray Bartolomé de las Casas & Ángeles Martín Mateo \\
Localidad & Sevilla & Alcalá de Guadaíra \\
\hline
\end{tabular}

Tabla 1. Características de la muestra 


\subsection{Contexto y participantes.}

La muestra estuvo compuesta por un total de 34 discentes de $3^{\circ}$ ciclo de Primaria divididos en dos grupos (Tabla 1).

Ambos centros comparten características socioculturales de acuerdo al ISC (Índice Social y Cultural), según los informes denominados «Indicadores homologados para la autoevaluación de los centros docentes públicos» de ambos centros educativos y del curso escolar 2011/2012 elaborados por la AGAEVE alcanzando un puntaje en valor en negativo, por debajo del valor «0». Por lo que podemos afirmar que nos encontramos con grupos de similares características.

\subsection{Instrumentos.}

\subsubsection{Pruebas de Competencia} Lingüística y criterios de corrección.

Para valorar el grado de adquisición de la competencia lingüística por parte de los participantes se utilizaron dos pruebas equivalentes, un pretest y un postest con la finalidad de evitar el posible efecto del recuerdo, conformadas por 15 ítems estandarizados y validados por la Consejería de Educación de la Junta de Andalucía (CEJA, 2011), extraídos de las «Pruebas de Evaluación de Diagnóstico» para Primaria sobre la competencia linguística. Los ítems elegidos para la elaboración de cada instrumento (pre y post) se ha realizado llevando un proceso de selección aleatoria de aquellos publicados por la CEJA. Su elaboración, según AGAEVE (2011) ha sido realizada por un grupo de personas expertas formado pro profesorado con suficiente experiencia docente en Educación Primaria e Inspectores de Educación especialistas.
Fueron tamizados por un pilotaje en una muestra conformada por 1000 alumnos para su valoración en relación a sus características psicométricas. Esta escala tiene un coeficiente Alfa de Cronbach: Escalado individual de 0,861 y escalado por centro educativo de 0,916 . En relación a los resultados de la fiabilidad podemos asegurar que la selección que se realiza sobre aquellos ítems más adecuados para mediar el nivel de competencia lingüística de los alumnos en primaria es fiable, superior a una coeficiente de 0.7

Las pruebas se corrigieron utilizando los criterios originales elaborados para estas pruebas, puntuando cada una de las 15 tareas entre 1 y 4 , siendo el 4 el mayor nivel de ejecución. Las preguntas incluidas en el estudio se agrupan $\mathbf{3}$ variables en base a tres subcompetencias de la competencia lingüística: «comprensión oral», «comprensión lectora» $\mathrm{y}$ «expresión escrita», objetivo de la investigación que hemos realizado.

\subsubsection{Programa de aplicación.}

El programa de aplicación a los dos grupos estuvo integrado por 2 unidades didácticas de Lengua Castellana y Literatura. La primera de ellas se implementó durante 15 sesiones de una hora de duración cada una de ellas, vertebrándose todos sus contenidos en relación al núcleo temático que denomina a la unidad; la segunda, a través de 10 sesiones de una hora de duración, y al igual que la anterior sigue el mismo modelo de interconexión de los contenidos.

\subsubsection{Recursos didácticos.}

El único aspecto que marcó la diferencia del programa de aplicación entre el grupo de 
control y el grupo experimental fue el uso de recursos basados en las TIC para el desarrollo de las tareas de enseñanza-aprendizaje. En ambos grupos, y de forma general, podemos destacar el uso de recursos didácticos tradicionales. Como recursos específicos, en el grupo experimental utilizaron: Pizarra Digital Interactiva, ultraportátil, reproductores digitales, conexión a Internet, aplicaciones interactivas y webs educativas.

\subsection{Procedimiento de la investigación.}

Antes de la aplicación del programa se procedió a realizar en ambos grupos, control y experimental, las medidas pretest de la variable «competencia linguística» utilizando el instrumento diseñado.

Posteriormente, se llevó a cabo el programa compuesto por dos unidades de trabajo del área de Lengua Castellana y Literatura, articuladas en 25 sesiones de unos 60 minutos de duración. Durante la implementación de las mismas, y solo con el grupo experimental se utilizaron herramientas TIC de forma activa, interactiva y dinámica. En el grupo de control, se evitó el uso de las mismas, utilizando solamente herramientas tradicionales.

Tras la finalización de las 25 sesiones, se procedió a realizar el postest, repitiendo la medida de la variable.

La información obtenida mediante los instrumentos de medición aplicados se volcó en una base de datos elaborada con el software SPSS versión 20.0. Para analizar y valorar los distintos datos recogidos se obtuvieron primero las puntuaciones medias en la dimensión de la Competencia Lingüística. Para comprobar la fiabilidad de este instrumento, se calculó la consistencia interna a través del coeficiente Alpha de
Cronbach. El valor obtenido ha sido superior a 0,80-mínimo recomendado por algunos autores (Henson, 2001). Posteriormente, para analizar la existencia o no de diferencias estadísticamente significativas entre las medidas del pretest y del postest, tanto en el grupo experimental como en el de control, se utilizó el estadístico T de Student para muestras relacionadas. Además, se llevó a cabo un análisis entre ambos grupos, experimental y de control, tanto en el pretest como en el postest, utilizando para ello la prueba $\mathrm{T}$ de Student para muestras independientes.

\section{Resultados.}

\subsection{Diferencias de partida entre el grupo experimental y el grupo control en los valores analizados (pretest).}

En primer lugar, con el objeto de determinar la igualdad entre grupos experimental y control previa a la intervención, se procedió a examinar y analizar la presencia o no de diferencias en el pretest entre el grupo de control y grupo experimental en relación a los valores analizados. Para determinar si había igualdad o no, se realizó en primer lugar la prueba de contraste de medias a través de la prueba $\mathrm{T}$ para muestras independientes. Previo al cálculo del valor $\mathrm{t}$, se valoró si la varianza entre los distintos grupos era igual o no, con el contraste de Levene sobre homogeneidad o igualdad de varianzas. El resultado del contraste es el que nos permitió decidir si podemos suponer que las varianzas poblaciones son iguales (si la probabilidad asociada al estadístico de Levene es mayor que 0,05) o distintas (si la probabilidad asociada al estadístico de Levene es menor que 0,05$)$. Tal y como se puede comprobar 
(ver Tabla 2), el análisis estadístico de las diferencias en las puntuaciones del pretest entre el grupo de control y el experimental, mostró respecto a la variable objeto de estudio que sí caben reseñar diferencias de partida ya que la comparativa de puntuaciones en el pretest nos muestra valores significativos en la media de la variable $(\mathrm{t}=2,506 ; \mathrm{p}=0,017)$ así como en su dimensión «Expresión escrita» $(\mathrm{t}=3,387 ; \mathrm{p}=$ $0,002)$, mientras que en las dimensiones referentes a la «comprensión escrita» y a «comprensión oral» no se presentaron diferentes. Si comparamos las medias, observamos que el grupo experimental presenta mayor valor de puntuación que el grupo de control en la el desarrollo de la competencia lingüística.

\begin{tabular}{|l|l|l|l|l|}
\hline VARIABLE & $\mathrm{t}$ & $\begin{array}{l}\text { Sig. } \\
\text { (bilateral) }\end{array}$ & $\begin{array}{l}\text { MEDIA } \\
\text {-grupo control- }\end{array}$ & $\begin{array}{l}\text { MEDIA } \\
\text {-grupo exp.- }\end{array}$ \\
\hline Comprensión oral &, 698 &, 490 & 2,1875 & 2,3056 \\
Comprensión escrita &, 895 &, 067 & 2,1875 & 2,6587 \\
Expresión escrita &, 387 &, $002^{*}$ & 1,5313 & 2,4167 \\
COMP. LINGÜÍSTICA &, 506 &, $017^{*}$ & 3,6438 & 2,4603 \\
\hline
\end{tabular}

*Nivel de significatividad $\leq=0,05$

Tabla 2. Resumen de la Prueba t de Student para muestras independientes de las diferencias pretest en el grupo de control y experimental.

\subsection{Diferencias entre el pretest y el postest en cada uno de los grupos (experimental y control).}

Para analizar si se habían producido diferencias significativas entre las puntuaciones postest-prestest pretest y postest, como consecuencia de la intervención, se realizó un análisis estadístico mediante la prueba t de Student para muestras relacionadas, encontrándose la existencia de las siguientes diferencias significativas entre las puntuaciones. Respecto al grupo de control, los resultados mostraron que no aparecieron diferencias significativas aunque sí cabe reseñar que los valores obtenidos en el postest sufren un descenso en relación a los obtenidos en el pretest. Respecto al análisis de datos recogidos del grupo experimental, sí podemos reseñar diferencias significativas entre los valores obtenidos en el pretest y postest en la variable «desarrollo de la competencia lingüística» $(t=5,257 ; \mathrm{p} \leq$ $0,001)$. Dado que las diferencias que se encontraron fueron muy significativas en esta última variable, se procedió al análisis de las distintas dimensiones que componen la misma, obteniendo grandes diferencias en «Comprensión escrita» $(\mathrm{t}=4,415 ; \mathrm{p} \leq 0,001)$ y «Comprensión Oral» $(\mathrm{t}=3,000 \mathrm{p}=, 008)$. Sin embargo, el grado de significatividad de la dimensión «Expresión Escrita» no es inferior a $\mathrm{p}=0,05$. 
4.3. Diferencias tras intervención entre el grupo experimental y el grupo control en los valores analizados (postest).

Por último, y con el objeto de comprobar la magnitud del cambio tras la implementación del programa, se llevó a cabo el análisis de los puntajes obtenidos en el postest comparando a los dos grupos (ver Tabla 3). Al igual que en el prestest, se procedió a determinar si había igualdad o no, realizándose de nuevo la prueba de contraste de medias. Anteriormente, se valoró si la varianza entre los distintos grupos era igual o no, con la finalidad de tener una decisión justificada del valor $\mathrm{t}$, y continuar realizando el estudio de datos a través de la prueba de Levene. Los resultados mostraron la existencia de diferencias estadísticamente significativas entre ambos grupos en la variable «competencia lingüística» $(\mathrm{t}=3,192$; $\mathrm{p}=, 004)$. En concreto, se obtuvieron resultados significativos en la dimensión 3 correspondiente a la «comprensión escrita» $(\mathrm{t}=2,711 ; \mathrm{p}=, 011)$ y la «expresión escrita» $(\mathrm{t}=3,634 ; \mathrm{p}=, 001)$. Cabe destacar que en todos los casos, los discentes que habían «sufrido» la inclusión de las TIC en la rutina escolar dentro del área de Lengua Castellana y Literatura, mostraron puntuaciones más altas que en relación a los puntajes obtenidos en el prestest, y en comparación con los discentes que formaban el grupo de control.

\section{Discusión.}

Una vez analizados los resultados del presente estudio, se puede concluir que, de forma parcial, se confirma la hipótesis de partida con la presente investigación: el uso de las TIC de forma integrada en la praxis educativa en el área de Lengua Castellana y Literatura en Primaria_provocaría un cambio en el rendimiento académico en la competencia lingüística de los discentes, manifestándose diferencias significativas en la variable objeto de estudio en la comparación de los grupos establecidos, alcanzando mejores resultados (puntajes más altos) aquellos y aquellas que conforman el grupo experimental. En este sentido, se pudo observar cómo, efectivamente, los sujetos del grupo

\begin{tabular}{|l|l|l|l|l|}
\hline VARIABLE & t & Sig. & MEDIA & MEDIA \\
& & (bilateral) & -grupo control- & -grupo exp.- \\
\hline Comprensión oral & 1,168 &, 251 & 2,2500 & 2,5913 \\
Comprensión escrita- & 2,711 &, $011^{*}$ & 2,2054 & 2,4861 \\
Expresión escrita- & 3,634 &, $001^{*}$ & 1,5938 & 2,8571 \\
COMP. LINGÜÍSTICA & 3,192 &, $004^{*}$ & 2,0164 & 2,4306 \\
\hline
\end{tabular}

*Nivel de significatividad $\leq=0,05$

Tabla 3. Resumen de la Prueba t de Student para muestras independientes de las diferencias postest en el grupo de control y experimental. 
experimental obtuvieron mejores resultados en el desarrollo de la competencia lingüística. Ambos grupos partieron de puntuaciones similares en dichas variables antes de empezar la intervención, ya que no se obtuvieron diferencias significativas de partida entre las puntuaciones pretest, tal y como se puede apreciar en sus dimensiones «comprensión oral» $\mathrm{y}$ «comprensión escrita». Pero una vez finalizada la implementación del programa en ambos grupos, se observaron diferencias significativas entre las puntuaciones postest del grupo control y experimental, de tal forma que se produjo un aumento significativo la competencia lingüística de los sujetos del grupo experimental en comparación con los sujetos del grupo control, pues se obtuvieron puntuaciones medias más altas en estas medidas en el grupo experimental en comparación con las puntuaciones del grupo control, y con un grado de significatividad reseñable en el plano estadístico.

Al realizar el análisis de las diferencias entre las puntuaciones postest-pretest en el grupo experimental, se observó la aparición de diferencias significativas entre ambas puntuaciones en las medidas de la variable «competencia lingüística» y en sus dimensiones «comprensión escrita», «comprensión oral». Tras ser evaluadas y analizadas las citadas, se detectó la obtención de un aumento significativo de las puntuaciones medias postest en comparación con las medias pretest en dichas variables. Sin embargo, no aparecieron diferencias significativas entre las medias de las puntuaciones postest y pretest en el grupo control para las diferentes puntuaciones de las distintas variables obtenidas, así como en sus bloques y dimensiones correspondientes. En todos los casos, las diferencias, ya sean de corte significativo estadístico o no, se dan siempre en una misma dirección: a favor del grupo que recibió el «tratamiento», el grupo experimental. Es decir, la inclusión de las TIC en las tareas de aprendizaje y en las actividades y acciones de enseñanza mejoró significativamente un mayor dominio de la comprensión lectora contribuyendo de forma importante y significativa en el desarrollo de la competencia lingüística. También mejoró las otras dimensiones de la competencia lingüística, aunque no de forma estadísticamente significativa.

Estos resultados concuerdan con los encontrados en otras investigaciones, donde se avala el uso de las TIC como herramienta efectiva para la competencia lingüística, produciendo mejoras significativas en su desarrollo, manifestada en evidencias como las tareas que componen los instrumentos diseñados para, en este estudio en concreto, su medición. En la misma línea, (Shapley, Sheehan, Maloney, \& Caranikas-walker, 2010), así como Marqués (2011) comprobaban que el uso de las TIC provoca mejores resultados en la realización de tareas relacionadas con el área de Lengua. Además, tal y como afirmaba (Penuel, 2006) las TIC provocan efectos en la dimensión expresión escrita de corte consistente y positivo. El hecho de que con las TIC en los procesos de enseñanzaaprendizaje se repliquen modelos exteriores más familiares, cómodos y factibles al sujeto, es una ventaja directa. Así pues, los resultados manifiestan que con su uso el dominio de la comprensión lectora aumenta significativamente, ya que dota, como hemos reseñado anteriormente de estrategias para actuar de forma más eficaz y eficiente ante la tarea de comprender un texto. En cambio, en la dimensión «expresión escrita» no aparece 
un grado de significatividad relevante, ya que posiblemente, para la contribución a su desarrollo, sean necesario no sólo acciones basadas en el modelado, sino las propias de la escuela tradicional al presentar en estas edades presentan un mayor dominio de la escritura de forma manual en relación a un dominio de la escritura mecanográfica digitalizada (con herramientas TIC), y por lo tanto, es más rápido y compensador, apresar con palabras el pensamiento a través de la técnica tradicional del lápiz y papel, que con el ordenador.

\section{Conclusiones.}

Tras la valoración de los resultados objetivos, creemos que el uso integrado de las TIC para el desarrollo de la competencia lingüística, favorece de forma incipiente la contribución al desarrollo de la misma, en la línea de investigaciones como (Area Moreira, 2005, 2010) que afirma en sus investigaciones que las TICs generan innovaciones a nivel de centro, a nivel de aula y del profesorado que las lleva a cabo.

Los estudiantes aprenden unos de otros, se desarrollan aprendizajes más dialógicos y constructivos, centrados en la negociación y el debate que en la transmisión. Los alumnos se ayudan para construir conjuntamente el conocimiento personal, con respeto a los otros y otras, escuchándose, valorando diversas perspectivas... La reciprocidad estudiante-TIC es totalmente recíproca. El discente planifica, interpreta, decide, evalúa la información y las TIC presentan, almacenan, clasifican, reproducen, etc. De esta manera se lleva cabo un ensayo más real, propio y significativo, con la realidad imperante, al estar conectado los patrones y herramientas de la escuela con los patrones que se llevan a cabo fuera de las aulas, potenciando así una mayor simbiosis entre la cultura escolar y la cultura social. Por consiguiente, y según los resultados obtenidos en el estudio realizado, es evidente que el uso de las TIC de forma integral y transversal aumenta el dominio de estrategias de aprendizaje en relación a la competencia lingüística, siempre que su inclusión en la praxis, esté dentro de una metodología que asimile modelos de investigación-acción (Marcelo, 2002; Oliver \& Corn, 2008). Hay que tener en cuenta que con la inclusión o modificación de un solo factor, como por ejemplo en este estudio, ciertas variables van a ser modificadas si se transforman los métodos o componentes del aprendizaje.

El principal aspecto que hay que tener en cuenta es que solamente con la nueva tecnología no se va a formar sujetos más creativos, que aprendan mejor lengua, trabajen mejor en equipo, etc. Yo diría que tendríamos que avanzar hacia una innovación radical, no incremental, hacia nuevas maneras de transmitir los conocimientos, nuevas formas de combinar distintas disciplinas, con nuevos puentes desde y hacia la educación... En fin, hemos repetido patrones que se han replicado, por lo menos, en los últimos $30 \mathrm{o}$ 40 años, de manera sistemática (Cobo Romaní, 2011, p. 27).

Por último, cabe señalar que aunque los resultados del presente trabajo de investigación han sido positivos, hay que mostrar cautela respecto a su generalización por dos razones argumentadas. En primer lugar, porque la muestra del estudio fue pequeña, y en segundo lugar, porque dentro del aula y de los procesos educativos siempre hay variables incontroladas o aspectos influyentes que alteran o contaminan los resultados. 
No obstante, y con la finalidad de elucubrar más en este ámbito, sería necesario llevar a cabo otros estudios para comprobar sus efectos en el tiempo, así como la indagación en elementos específicos que interfieren no sólo en los procesos de aprendizaje, sino en los procesos de enseñanza.

\section{Referencias bibliográficas}

Alonso Cano, C., Romeu Fontanillas, T., \& Guitert Catasús, M. (2014). Los Entornos 1x1 en Cataluña. Entre las expectativas de las políticas educativas y las voces del profesorado. Educar, 50 (1), 41-64. Recuperado de http://ddd.uab.cat/record/ 115076/\%5Cnhttp://ddd.uab.cat/pub/educar/ educar_a2014m1-6v50n1/educar_a2014m16v50n1p41.pdf

Antón, J. (2011). Cristóbal Cobo Romaní: cambian las tecnologías, pero se replican los patrones y los errores del pasado. Cuadernos de Pedagogía, (418), 26-29.

Area Moreira, M. (2005). Las tecnologías de la información y comunicación en el sistema escolar. Una revisión de las lineas de investigación. Revista Electrónica de Investigación Y Evaluación Educativa, 11(1), 3-25. Recuperado de http://www.uv.es/ RELIEVE/v11n1/RELIEVEv11n1_1.htm

Area Moreira, M. (2010). El proceso de integración y uso pedagógico de las TIC en los centros educativos. Revista de Educación, 352, 77-97. Recuperado de http:/ /redined.mecd.gob.es/xmlui/bitstream/ $\mathrm{h}$ a n d l e / $11162 / 79368 /$ 00820103009645.pdf?sequence $=1$

Cassany, D. (2005). Los significados de la comprensión crítica. Lectura Y Vida, 26(3), 32-45. Recuperado de http:// www.lecturayvida.fahce.unlp.edu.ar/ numeros/a26n3/26_03_Cassany.pdf
Cassany, D. (2008). La lectura ciudadana. La Lectura En España. Informe 2008, 225243. Recuperado de http://repositori.upf.edu/ handle/10230/21218

Coll, C. (2009). Aprender y enseñar con las TIC: expectativas, realidad y potencialidades. Universia, 1.

Decreto 97/2015, de 3 de marzo, por el que se establece la ordenación y las enseñanzas correspondientes a la Educación primaria en Andalucía. Boletín Oficial de la Junta de Andalucía. Sevilla, 13 de marzo de 2015, núm. 50, pp. 11-22. Recuperado de http:// www.juntadeandalucia.es/boja/2015/50/1

García Ruiz, R., Ramírez García, A., \& Rodríguez Rosell, M. del M. (2014). Educación en alfabetización mediática para una nueva ciudadanía prosumidora. Comunicar: Revista Científica Iberoamericana de Comunicación Y Educación. https://doi.org/10.3916/C432014-01

Gracida, I., \& Lomas, C. (2011). Textos e hipertextos. Textos de Didáctica de la Lengua y la Literatura, (57), 5-11.

Henson, R. K. (2001). Understanding Internal Consistency Reliability Estimates: A Conceptual Primer on Coefficient Alpha. Measurement \& Evaluation in Counseling \& Development (American Counseling Association). Retrieved from http:// $\mathrm{s}$ e a r h . e b s c o hos t. co m/ log i n a s p ? d i rect $=$ true $\& d$ b $=$ pbh\&AN $=5542030 \&$ site $=$ ehost-live

Himle, M. B., \& Woods, D. W. (2005). An experimental evaluation of tic suppression and the tic rebound effect. Behaviour Research and Therapy, 43(11), 1443-1451. https://doi.org/10.1016/j.brat.2004.11.002

Junta de Andalucía. Consejería de Educación. (2007). Marco Teórico. Evaluación de Diagnóstico 2006-2007. 
Junta de Andalucía. (2011) Evaluación de diagnóstico 2010-2011: Informe de resultados. Recuperado de http:// www.juntadeandalucia.es/educacion/ agaeve/docs/05_Informe_PED_2010-11.pdf

Ley 17/2007, de 10 de diciembre, de Educación de Andalucía. Boletín Oficial de la Junta de Andalucía. Sevilla, 26 de diciembre de 2007, núm. 252, pp. 5-35. Recuperado de http:// www.juntadeandalucia.es/boja/2007/252/1

Macau, R. (2004). TIC/: ¿PARA QUÉ? (Funciones de las tecnologías de la información y la comunicación en las organizaciones). Revista de Universidad $Y$ Sociedad Del Conocimiento, vol 1 numl(ISSN 1234-5678), 1-12. Recuperado de h t t p : / w w w.uoc.edu/rusc/dt/esp/ macau0704.pdf\%5Cnhttp://www.uoc.edu/ rusc/dt/esp/macau0704.html

Marcelo, C. (2002). Los profesores como trabajadores del conocimiento: Certidumbres y desafíos para una formación a lo largo de la vida. Educar, 27-56. Recuperado de http:// dialnet.unirioja.es.biblioremot.uvic.cat/ servlet/articulo? $\operatorname{codigo}=440774 \&$ info $=r$ esumen\&idioma $=\mathrm{ENG}$

Maria Mama, T., \& Hennessy, S. (2013). Developing a typology of teacher beliefs and practices concerning classroom use of ICT. Computers and Education, 68, 380-387. h t t p s : / / d o i.org/10.1016/ j.compedu.2013.05.022

Mariscal, S., López-Ornat, S., Gallego, C., Gallo, P., Karousou, A., \& Martínez, M. (2007). La evaluación del desarrollo comunicativo y linguiístico mediante la versión española de los inventarios MacArthur-Bates. Psicothema, 19(2), 190-197.

Nardocci, I. M., \& Nascimento, J. V. (2012). A manifestação do ethos pelas marcas de modalidade no discurso de fóruns educacionais digitais. Calidoscópio, 10(3), 270-277. https://doi.org/10.4013/3112

OECD. (2015). Students, Computers and Learning: Making the Connection, PISA, OECD Publishing. OECD Publishing. https:/ /doi.org/10.1787/9789264239555-en

Oliver, K. M., \& Corn, J. O. (2008). Studentreported differences in technology use and skills after the implementation of one-to-one computing. Educational Media International, 45(3), 215-229. https://doi.org/ $10.1080 / 09523980802284333$

Orden de 17 de marzo de 2015, por la que se desarrolla el currículo correspondiente a la Educación Primaria en Andalucía. Boletín Oficial de la Junta de Andalucía. Sevilla, 27 de marzo de 2015, núm. 60, pp. 9-696. Recuperado de http:// www.juntadeandalucia.es/boja/2015/60/1

Penuel, W. R. (2006). Implementation and effects of one-to-one computing initiatives: A research synthesis. Journal of Research on Technology in Education, 38(3), 329-348. https://doi.org/1.541.302.3777

Ramírez Leyva, E. (2001). La lectura en la sociedad contemporánea. Investigación Bibliotecológica, 15(30), 114-131. Recuperado de http://www.ejournal.unam.mx/ ibi/vol15-30/IBI03006.pdf

Ramírez, R., Renés, P., \& Delgado,A. (2014). La competencia mediática y el área de lengua castellana y literatura de educación primaria. Análisis curricular en España. Calidoscópio, 12(3), 345-355. Recuperado de http:// s e a r c h.proquest.com/openview/ 619ec84598dfd031c949ae5755232e61/1?pqorigsite $=$ gscholar $\&$ cbl $=2040964$

Santiago Campión, R., Navaridas Nalda, F., \& Repáraz Abaitua, C. (2013). La escuela 2.0: reflexiones en torno a su eficacia en los centros educativos de La Rioja. Educación 
XX1, 17(1), 243-270. https://doi.org/10.5944/ educxx1.17.1.10713

Shapley, K. S., Sheehan, D., Maloney, C., \& Caranikas-walker, F. (2010). Evaluating the Implementation Fidelity of Technology Immersion and its Relationship with Student Achievement. The Journal of Technology, Learning, and Assessment, 9(4), 1-69.

Underwood, J. (2011). ¿La tecnología es capaz de transformar el leviatán educativo? Cuadernos de Pedagogía, (418), 16-19.

Fecha de recepción: 30-04-2017

Fecha de evaluación: 14-06-2017

Fecha de aceptación: 10-07-2017

Píxel-Bit. Revista de Medios y Educación. No 53 Julio 2018. ISSN: 1133-8482. e-ISSN: 2171-7966. doi: http://dx.doi.org/10.12795/pixelbit.2018.i53.08 